\title{
Papers
}

\section{Risk of subarachnoid haemorrhage in first degree relatives of patients with subarachnoid haemorrhage: follow up study based on national registries in Denmark}

David Gaist, Michael Væth, Ioannis Tsiropoulos, Kaare Christensen, Elisabeth Corder, Jørn Olsen, Henrik Toft Sørensen

\begin{abstract}
Objective To estimate the risk of occurrence of subarachnoid haemorrhage in first degree relatives (parents, siblings, children) of patients with subarachnoid haemorrhage.

Design Population based cohort study using data from the Danish National Discharge Registry and the Central Person Registry.

Subjects Incident cases of subarachnoid haemorrhage admitted to hospital from 1977 to 1995 (9367 patients) and their first degree relatives (14 781). Main outcome measures The incidence rate of subarachnoid haemorrhage was determined for the relatives and compared with that of the entire population, standardised for age, sex, and calendar period. This process was repeated for patients discharged from neurosurgery units, as diagnoses from these wards had high validity (93\%).

Results 18 patients had a total of 19 first degree relatives with subarachnoid haemorrhage during the study period, corresponding to a standardised incidence ratio of 2.9 (95\% confidence interval 1.9 to 4.6). Patients discharged from neurosurgery wards had a higher standardised incidence ratio $(4.5,2.7$ to 7.3).

Conclusions First degree relatives of patients with subarachnoid haemorrhage have a threefold to fivefold increased risk of subarachnoid haemorrhage compared with the general population.
\end{abstract}

\section{Introduction}

Subarachnoid haemorrhage, in $80 \%-90 \%$ of cases due to rupture of an intracranial aneurysm, occurs at all ages but strikes primarily in middle age, often with devastating consequences: about $40 \%$ of patients admitted to hospital die within 1 month and more than one third of survivors have severe disability. ${ }^{1}$ Several studies have indicated that smoking, hypertension, and alcohol misuse are important risk factors for subarachnoid haemorrhage due to aneurysms. ${ }^{2}$ Genetic factors may also have a role as close relatives harbour intracerebral aneurysms more frequently than the background population. ${ }^{3}$ Furthermore, intracerebral aneurysms are associated with several rare hereditary disorders-for example, polycystic kidney disease and Ehlers-Danlos syndrome type IV. ${ }^{4}$

Four recent studies indicate that first degree relatives (parents, full siblings, children) are at increased risk of subarachnoid haemorrhage..$^{5-8}$ In all but one of the existing studies, information on pedigrees and identification of cases among relatives were based on self reported or proxy reported data, which can be subject to selection and recall biases making quantitative estimates inaccurate. We believe that creation of pedigree and case identification by means of population based registries provide less opportunity for selection and avoid recall bias. We therefore used nationwide registries to estimate the incidence rate of subarachnoid haemorrhage in first degree relatives of patients with the disorder and to compare it with the rate in the Danish population.

\section{Methods}

\section{Nationwide registries}

We performed a follow up study based on information from two nationwide registries: the Danish National Discharge Registry and the Danish Central Person Registry. We identified patients with subarachnoid haemorrhage in the discharge registry and identified their first degree relatives in the central registry. Information on essentially all (99.4\%) discharges from Danish hospitals has been recorded in the discharge registry since $1977 .{ }^{9}$ Recorded data include the dates of admission and discharge, up to 20 discharge diagnoses, hospital and department codes, and the surgical procedures performed. Discharge diagnoses were classified according to the Danish version of the ICD-8 (international classification of diseases, 8th revision) for 1977 to 1993 and the ICD-10 (10th revision) for 1994-5. A 10 digit civil registration number, which is permanent and unique to each Danish citizen, is also recorded, enabling the compilation of patient specific discharge histories. Data were retrieved on all persons listed with a diagnosis of subarachnoid haemorrhage (ICD-8 codes 430; ICD-10 codes I60) in the discharge registry from 1 January 1977 to 31 December 1995. We identified index episodes, defined as the first registered admission to hospital with a diagnosis of subarachnoid

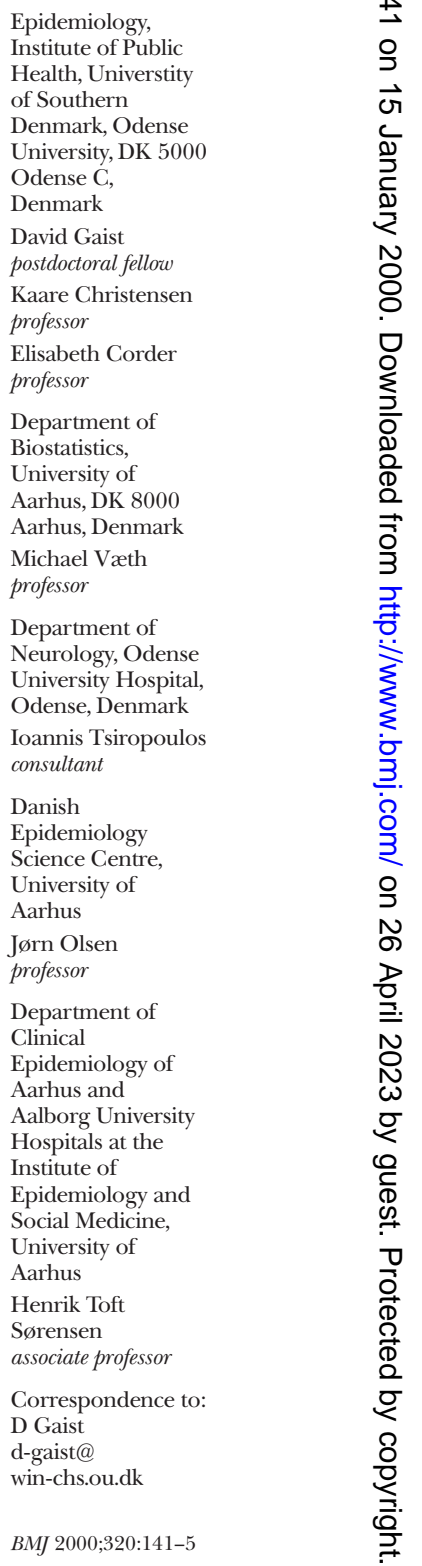


haemorrhage within the study period. Coding of the index episode as a recurrent or previous episode of subarachnoid haemorrhage led to exclusion. We also excluded patients with concurrent codes for cerebral arteriovenous malformations (ICD-8 codes 430.01 and 430.91; ICD-10 codes Q28.2 and Q28.3). In Denmark, suspicion of subarachnoid haemorrhage usually leads to rapid transfer of patients to neurosurgery or neurology departments for more advanced diagnostic investigation and treatment. We therefore classified patients according to the most specialised department they had been admitted to or transferred to within 21 days of their first admission with diagnosed subarachnoid haemorrhage: neurosurgery (most specialised), neurology, or "other department" (least specialised) according to the register data.

Since 1 April 1968 all persons residing in Denmark have been registered in the Danish Central Person Registry with their civil registration number, date of birth, residency, date of immigration or emigration, and date of death. For each individual the civil registration numbers of the parents are also recorded. The coverage of information on the parental civil registration numbers, however, increased over time from less than 10\% for cohorts born before 1952 to $43 \%$ in the 1952 cohort, $96 \%$ in the 1959 cohort, and 99\% for cohorts born after $1970 .{ }^{10}$ Adopted children cannot be identified as such in the registry and are recorded under their adopted parents, but the magnitude of this misclassification is small. ${ }^{10}$

We retrieved data on vital status from the registry for the period 1 April 1968 to 31 December 1995 for members of the subarachnoid haemorrhage inception cohort and their first degree relatives who could be traced in the central registry. For each group of first degree relatives, the person discharged with a diagnosis of subarachnoid haemorrhage on the earliest date, designated the index case, was identified. First degree relatives living in Denmark on the date of admission of the index case were followed up as of that date and until the first admission for subarachnoid haemorrhage, emigration, death, or end of study period, whichever event came first. Incident cases of subarachnoid haemorrhage that occured among first degree relatives during follow up were designated first degree relative cases.

\section{Validity of the diagnosis}

We studied the validity of a register diagnosis of subarachnoid haemorrhage in a sample from hospitals in Funen County (465 000 inhabitants; 9\% of the Danish population). Only hospitals with more than 10 registered patients in the study period were used-that is, a university hospital with a neurosurgery and a neurology department, and two other hospitals. Discharge records on 210 patients were retrieved for review. Data from discharge abstracts, medical records, or autopsy reports were abstracted to a structured form and evaluated, blinded with respect to family relationship, by an experienced neurologist (IT) according to predefined criteria. A register diagnosis of subarachnoid haemorrhage was accepted when a typical clinical history and at least one of the following were described: computed tomography findings compatible with blood in the subarachnoid space, xanthochromic cerebrospinal fluid, or subarachnoid haemorrhage or aneurysm established by angiography or autopsy.
Medical records could be traced in all but one case. All cases marked as recurrent or previous episodes of subarachnoid haemorrhage in the register (18 patients) were also recorded as such in the medical documents. In the remaining 191 cases the positive predictive value of a register diagnosis of subarachnoid haemorrhage was $93 \%$ (95\% confidence interval $85 \%$ to $98 \%$ ) for patients discharged from the neurosurgery department and $75 \%(60 \%$ to $87 \%)$ for patients discharged from the neurology department. For patients discharged from other non-specialty wards, the predictive value of a register diagnosis of subarachnoid haemorrhage was low: $47 \%$ (36\% to $59 \%$ ). We also validated the register diagnosis of subarachnoid haemorrhage for patients from families with more than one case of subarachnoid haemorrhage identified in the nationwide material ( 37 patients). The predictive value of a register diagnosis of subarachnoid haemorrhage in these cases, which were mostly admitted to neurosurgery wards, was $92 \%$ ( $78 \%$ to $98 \%)$. Overall the misclassification may thus be less than $20 \%$.

Familial subarachnoid haemorrhage is reported to be associated with congenital heart disease ${ }^{11}$ and several rare hereditary disorders, such as polycystic kidney disease and Ehlers-Danlos syndrome, ${ }^{4}$ which we wanted to exclude from the study. The retrieved medical records made no mention of such disorders. Furthermore, for the 37 familial cases we scanned all discharges in the register under any diagnosis for these persons and found no discharges with diagnostic codes corresponding to the above mentioned disorders.

\section{Statistical analyses}

We calculated age specific and sex specific incidence rates for the entire Danish population for each of the periods 1977-81, 1982-6, 1987-91, and 1992-5. Twenty age categories ( 5 year intervals) were used. Appropriate population denominators (person years at risk) were derived from annual population counts (1 year age categories for each sex) obtained from Statistics Denmark. ${ }^{12}$ To study the incidence rate of subarachnoid haemorrhage in first degree relatives the follow up data were aggregated in a multiway person year table. The main table was based on sex, age (5 year intervals), and calendar period (four periods). To study the effect of follow up time (time since admission of index case categorised as less than 1 year, 1-7 years, and more than 7 years), type of relative (parent, sibling, child), and the sex of the index case, three additional person year tables were made based on each of these factors and including age, sex, and calendar period. The table including sex of the index case was restricted to children of the index cases.

Time at risk for first degree relatives was calculated from the date of admission of the index case until the first admission for subarachnoid haemorrhage, emigration, death, or end of study period, whichever event came first. The incidence rate of subarachnoid haemorrhage in first degree relatives of patients with subarachnoid haemorrhage was compared with that of the entire population using a Poisson regression model. The results were summarised by standardised incidence ratios and 95\% confidence intervals. ${ }^{13}$ The main table was used to assess the effect of sex, age, and calendar period. These factors were examined one at a time because the limited number of cases did not allow 
a comprehensive analysis of interactions. The additional tables were used to evaluate the effects of follow up time, type of relative, and sex of index case. To study the effect of potential misclassification-that is, labelling registered cases (which in fact were prevalent cases) as incident cases, we successively restricted the study period by letting it begin in 1982, 1987, and 1992.

Misclassification of diagnoses in the discharge registry results in inclusion of non-cases in the analyses, which leads to an underestimate of the relative risk. Neurosurgery units had the lowest proportion of misclassification of cases according to our validation study. We therefore repeated all analyses after restricting the sample to patients (including index cases and first degree relative cases) discharged from neurosurgery units. The study was approved by all seven regional ethics committees and the Danish registry board.

\section{Results}

We identified 9367 patients admitted to hospital for the first time with subarachnoid haemorrhage during the study period. Patients were more frequently female and middle aged, and the 30 day case fatality rate was high (table 1). In all, $77 \%$ (7200 patients) of patients had been admitted, or transferred, to neurospecialty units. By comparison, cases with identified first degree relatives were younger (mean difference 4.8 years) and were more frequently admitted to departments with neurospecialty $(84 \% ; 5177$ patients) (table 1$)$. According to our data, the overall incidence of subarachnoid haemorrhage in Denmark in 1986 (the mid period of the study) was 9.25 per 100000 person years. Age specific incidence rates were stable during most of the study period and peaked in the fifth and sixth age decade for both sexes (results not shown).

First degree relatives could be traced for 6175 cases (66\%). We identified 14781 relatives of the index cases, mostly offspring, who were followed for a total of 108933 person years (table 2). Eighteen index cases had a total of 19 first degree relatives (10 women and nine men) who had had a subarachnoid haemorrhage during follow up, 16 of whom were admitted to neurosurgery units. The median age at admission to hospital was 33.7 years (range 19.0-61.2 years). Nine of the 19 subjects (47\%) died within 30 days of admission to hospital.

First degree relatives of patients with subarachnoid haemorrhage had a threefold risk of developing this condition compared with the general population. Restricting the analysis to cases admitted to neuro-
Table 1 Characteristics of patients with subarachnoid haemorrhage in Denmark 1977-95. Values are numbers (percentages) unless indicated otherwise

\begin{tabular}{|c|c|c|}
\hline Characteristic & $\begin{array}{l}\text { All cases } \\
(\mathrm{n}=9367)\end{array}$ & $\begin{array}{l}\text { Cases with traced first } \\
\text { degree relatives ( } n=6175)\end{array}$ \\
\hline \multicolumn{3}{|l|}{ Sex: } \\
\hline Female & $5466(58.4)$ & $3503(56.7)$ \\
\hline Male & $3901(41.6)$ & $2672(43.3)$ \\
\hline \multicolumn{3}{|l|}{ Age at index admission to hospital (years) ${ }^{*}$ : } \\
\hline$<40$ & $1844(19.7)$ & $1683(27.3)$ \\
\hline $40-59$ & $4190(44.7)$ & $3347(54.2)$ \\
\hline $60-79$ & $2995(32.0)$ & 1125 (18.2) \\
\hline$\geqslant 80$ & $338(3.6)$ & $20(0.3)$ \\
\hline Mean age at index admission to hospital (years) ${ }^{*}$ & 53.5 & 47.7 \\
\hline \multicolumn{3}{|l|}{ Type of department†: } \\
\hline Neurosurgery & $6292(67.2)$ & $4688(75.9)$ \\
\hline Neurology & $908(9.7)$ & $489(7.9)$ \\
\hline Other & $2167(23.1)$ & $998(16.2)$ \\
\hline $\begin{array}{l}\text { Case fatality rate within } 30 \text { days from index admission to } \\
\text { hospital } \neq\end{array}$ & $3560(38.0)$ & $2168(35.1)$ \\
\hline
\end{tabular}

${ }^{*}$ First registered admission to hospital with diagnosis of subarachnoid haemorrhage in study period. †Most specialised department admitted, or transferred, to within 21 days of index admission to hospital. fEight patients not included owing to missing information on vital status.

surgery wards increased the standardised incidence ratio to 4.5 (2.7 to 7.3). Left truncating the time window of case ascertainment and follow up had very little effect on these results (table 3). In the analysis both of all cases and of cases admitted to neurosurgery units, the excess risk did not depend on sex $(\mathrm{P}>0.5$ in both analyses), age (test for trend, $\mathrm{P}>0.5$ and $\mathrm{P}=0.2$ respectively), or calendar period ( $\mathrm{P}=0.2$ in both analyses)

A tendency for the standardised incidence ratio to decrease with increasing follow up time was not statistically significant (all cases: 11\% per year of follow up, $\mathrm{P}=0.1$; neurosurgery cases: $8 \%$ per year of follow up, $\mathrm{P}=0.2$ ). The standardised incidence ratio showed some variation between types of relative (highest among children), but the tendency was not statistically significant (all cases: $\mathrm{P}>0.5$; neurosurgery cases: $\mathrm{P}=0.4$ ) (table 2). Finally, the sex of the index case did not have any significant impact on the excess risk of the children (all cases: $\mathrm{P}=0.5$; neurosurgery cases: $\mathrm{P}=0.4$ ).

\section{Discussion}

We found that first degree relatives of patients who had a subarachnoid haemorrhage were at a threefold to fivefold increased risk of subarachnoid haemorrhage compared with the background population. The main strengths of the present study are its large size, and the setting-a nationwide uniformly organised health

Table 2 Incidence rate of subarachnoid haemorrhage in first degree relatives of index cases*

\begin{tabular}{|c|c|c|c|c|c|c|}
\hline $\begin{array}{l}\text { Relationship to } \\
\text { index case }\end{array}$ & $\begin{array}{l}\text { Type of hospital } \\
\text { ward where cases } \\
\text { were identified }\end{array}$ & $\begin{array}{l}\text { No of first degree } \\
\text { relatives identified }\end{array}$ & $\begin{array}{l}\text { Mean age at start of } \\
\text { follow upł (years) }\end{array}$ & $\begin{array}{l}\text { Person years of } \\
\text { follow up } \ddagger\end{array}$ & $\begin{array}{l}\text { Incident cases in first } \\
\text { degree relatives }\end{array}$ & $\begin{array}{l}\text { Standardised incidence } \\
\text { ratio (95\% confidence } \\
\text { interval) }\end{array}$ \\
\hline \multirow[t]{2}{*}{ Child } & Any & 11640 & 20.8 & 88989 & 13 & $3.5(2.0-6.0)$ \\
\hline & Neurosurgical $\dagger$ & 9093 & 20.6 & 69314 & 12 & $5.7(3.3-10.1)$ \\
\hline \multirow[t]{2}{*}{ Parent } & Any & 1720 & 54.5 & 10881 & 5 & $2.3(0.9-5.4)$ \\
\hline & Neurosurgical† & 1188 & 56.1 & 7453 & 3 & $2.5(0.8-7.9)$ \\
\hline \multirow[t]{2}{*}{ Sibling } & Any & 1421 & 26.2 & 9063 & 1 & $2.0(0.3-14.0)$ \\
\hline & Neurosurgical† & 1014 & 27.7 & 6528 & 1 & $3.3(0.5-23.5)$ \\
\hline \multirow[t]{2}{*}{ Total } & Any & 14781 & 25.3 & 108933 & 19 & $2.9(1.9-4.6)$ \\
\hline & Neurosurgical† & 11295 & 25.0 & 83295 & 16 & $4.5(2.7-7.3)$ \\
\hline
\end{tabular}

*Person first diagnosed (in calendar time) with subarachnoid haemorrhage in family.

†Relatives contributed person years of follow up from date of admission to hospital of index cases.

†Only first degree relatives of index cases admitted to neurosurgery wards included. Among these first degree relatives, incident cases of subarachnoid haemorrhage occurring during follow up were only counted if admitted to a neurosurgery ward. 
Table 3 Standardised incidence ratio (95\% confidence intervals) of subarachnoid haemorrhage in first degree relatives of index cases ${ }^{*}$

\begin{tabular}{lcc} 
& \multicolumn{2}{c}{ Type of hospital ward } \\
\cline { 2 - 3 } Period & Any & Neurosurgeryt \\
\hline All years (1977-95) & $2.9(1.9$ to 4.6$)$ & $4.5(2.7$ to 7.3$)$ \\
\hline $1982-95$ & $2.7(1.7$ to 4.3$)$ & $4.3(2.6$ to 7.1$)$ \\
\hline $1987-95$ & $2.9(1.8$ to 4.7$)$ & $4.8(2.9$ to 8.0$)$ \\
\hline $1992-5$ & $2.2(1.1$ to 4.5$)$ & $4.1(2.0$ to 8.2$)$
\end{tabular}

*Person first diagnosed (in calendar time) with subarachnoid haemorrhage in family.

tBoth index case and first degree relatives were admitted to neurosurgery ward.

system with free access to medical care. Furthermore, our study was not vulnerable to the potential bias in studies using self reported or proxy reported data-that is, that relatives of patients with subarachnoid haemorrhage participate and report differently, depending on the number of cases in the family.

Our results are, however, in line with previous studies using different designs and populations. ${ }^{5-8}$ In a case-control study of 149 patients with subarachnoid haemorrhage, cases were 1.8 times more likely than controls to report having first degree relatives with subarachnoid haemorrhage. ${ }^{5}$ Bromberg et al interviewed relatives of 163 cases of subarachnoid haemorrhage that were admitted to hospital. ${ }^{6}$ Ten cases of subarachnoid haemorrhage in first degree and four in second degree relatives could be verified by review of medical records, corresponding to a relative risk of 6.6. ${ }^{6}$ In another study, 11 first degree relatives of 76 patients with subarachnoid haemorrhage had had an aneurysmal subarachnoid haemorrhage, which corresponded to a relative risk of 4.1 compared with the background population. ${ }^{7}$ De Braekeleer et al traced all 533 cases of subarachnoid haemorrhage and unruptured intracranial aneurysms through hospital registries, and matched controls were identified through a population register in an isolated population with a high degree of inbreeding. ${ }^{8}$ Cases were 4.7 times more likely to have affected first degree relatives than were controls. However, the interpretation of the findings of this study is hampered by the inclusion of patients with unruptured aneurysms in the case group.

Familial cases of intracranial aneurysm have been reported to occur, with a slight preponderance in women, at a younger age, and with similar severity and case fatality as non-familial cases. ${ }^{14-19}$ Our estimate of the relative risk of subarachnoid haemorrhage in first degree relatives of patients with the disorder was not influenced by sex, age, calendar period, follow up, or the sex of the index case. However, this could be owing to the small number of familial cases of subarachnoid haemorrhage in our study.

The low incidence rate and high 30 day case fatality rate of subarachnoid haemorrhage in Denmark are comparable to results reported in recent studies. ${ }^{20-22}$ In contrast to previous studies reporting a decrease in subarachnoid haemorrhage incidence over time, ${ }^{20-23}$ we found that the incidence rate was stable for most of the study period.

\section{Potential limitations of the study}

Our study has several potential weaknesses. Prevalent cases, surviving a first admission to hospital for subarachnoid haemorrhage before 1977, could be misclassified as incident cases if admitted for a recurrent episode of subarachnoid haemorrhage during the study period. We addressed this problem by excluding patients with a register code at the index admission to hospital, indicating a recurrent episode. The high case fatality rate and the robustness of our results to progressively left truncating the time window indicate that the extent of the problem is minor.

The coverage of family relations by the central registry increased over time, which could affect our results. However, restricting the material to families identified during the period with essentially full coverage by the central registry had little influence on the estimates (standardised incidence ratio 3.0). The identified first degree relatives were mainly offspring and often did not reach the age at which subarachnoid haemorrhage incidence peaks during follow up. This may result in an underestimate of the relative risk of subarachnoid haemorrhage in first degree relatives, in particular for siblings, which have been reported to be at a higher risk than other first degree relatives. ${ }^{7}$ Longer follow up of the cohort will disclose whether this is the case. The registration of adopted children under their nonbiological parents in our data also tends to dilute the observed association, but the magnitude of this problem is small. ${ }^{10}$

It is conceivable that relatives of patients with subarachnoid haemorrhage would have a lower threshold for seeking medical help if they experience symptoms that could be attributed to subarachnoid haemorrhage. Review of the discharge records and the high case fatality rate observed in this group preclude this. Our study did not include patients with subarachnoid haemorrhage who died before admission to hospital. This, however, happens only in some $11 \%$ to $14 \%$ of all patients with subarachnoid haemorrhage. ${ }^{18}$ Familial cases have not been reported to be more likely to belong to this group.

\section{Conclusion}

Our study, which reduced selection bias and was not prone to recall bias, leads us to conclude that first degree relatives of patients with subarachnoid haemor-

\section{What is known on this topic}

Several observational studies have indicated that first degree relatives of patients with subarachnoid haemorrhage are at increased risk of having this disorder. However, the validity of the risk estimates could be questioned owing to potential problems of selection and recall bias

\section{What this paper adds}

This follow up study overcame some of these problems by using national registries in Denmark to create pedigrees and to identify incident cases of subarachnoid haemorrhage

The study confirmed that first degree relatives of patients with subarachnoid haemorrhage are at a threefold to fivefold increased risk of experiencing a subarachnoid haemorrhage compared with the general population, but the incidence rate of subarachnoid haemorrhage is low 
rhage are at a threefold to fivefold increased risk of having a subarachnoid haemorrhage.

Contributors: DG, KC, and HTS initiated the study. DG was principal investigator and study coordinator, contributed to the design of the study, the collection and the analysis of the data, and wrote the initial draft; he will act as guarantor for the paper. MV contributed to the design of the study and did the statistical analyses. IT contributed to the design of the validation part of the study, which he also conducted. KC, EC, JO, and HTS participated in the design and discussed core ideas. All authors contributed to the interpretation of the data and the writing of the paper.

Funding: This study was funded by a grant from Sygekassernes Helsefond (No 11/222-96). The activities of the Danish Epidemiology Science Centre are financed by a grant from the National Danish Research Foundation. Competing interests: None declared.

1 Schievink WI. Intracranial aneurysms. N Engl J Med 1997;336:28-40.

2 Teunissen LL, Rinkel GJ, Algra A, van-Gijn J. Risk factors for subarachnoid hemorrhage: a systematic review. Stroke 1996;27:544-9.

3 Ronkainen A, Hernesniemi J, Puranen M, Niemitukia L, Vanninen R, Ryynanen M, et al. Familial intracranial aneurysms. Lance 1997:349:380-4.

4 Schievink WI. Genetics of intracranial aneurysms. Neurosurgery 1997;40:651-62.

5 Wang PS, Longstreth-WTJ, Koepsell TD. Subarachnoid hemorrhage and family history. A population-based case-control study. Arch Neurol 1995;52:202-4.

6 Bromberg JE, Rinkel GJ, Algra A, Greebe P, van-Duyn CM, Hasan D, et al. Subarachnoid haemorrhage in first and second degree relatives of patients with subarachnoid haemorrhage. BMJ 1995;311:288-9.

7 Schievink WI, Schaid DJ, Michels VV, Piepgras DG. Familial aneurysmal subarachnoid hemorrhage: a community-based study. J Neurosurg 1995;83:426-9.

8 De-Braekeleer M, Perusse L, Cantin L, Bouchard JM, Mathieu J. A study of inbreeding and kinship in intracranial aneurysms in the Saguenay
Lac-Saint-Jean region (Quebec, Canada). Ann Hum Genet 1996;60: 99-104

9 Sundhedsstyrelsen. Aktiviteten $i$ sygehusvasenet 1979. Copenhagen, Denmark: Sygehusstatistik, Sundhedsstyrelsen, 1981.

10 Christensen K, Schmidt MM, Vaeth M, Olsen J. Absence of an environmental effect on the recurrence of facial-cleft defects. N EnglJ Med 1995;333:161-4

11 Schievink WI, Mokri B, Piepgras DG, Gittenberger-de Groot CE. Intracranial aneurysms and cervicocephalic arterial dissections associated with congenital heart disease. Neurosurgery 1996;39:685-90.

12 Statistics Denmark. Statistical yearbook 1998. Aarhus: Statistics Denmark, 1998

13 Breslow NE, Day NE. Statistical methods in cancer research. The design and analysis of cohort studies. Oxford: Oxford University Press, 1987.

14 Nörrgård Ö, Ängquist K-A, Fodstad H, Forsell Å, Lindberg M. Intracranial aneurysms and heredity. Neurosurgery 1987;20:236-9.

15 Bromberg JE, Rinkel GJ, Algra A, Limburg M, van-Gijn J. Outcome in familial subarachnoid hemorrhage. Stroke 1995;26:961-3.

16 Leblanc R, Melanson D, Tampieri D, Guttmann RD. Familial cerebral aneurysms: a study of 13 families. Neurosurgery 1995;37:633-8.

17 Ronkainen A, Hernesniemi J, Tromp G. Special features of familial intracranial aneurysms: report of 215 familial aneurysms. Neurosurgery 1995:37:43-6.

18 Schievink WI, Wijdicks EF, Parisi JE, Piepgras DG, Whisnant JP. Sudden death from aneurysmal subarachnoid hemorrhage. Neurology 1995;45:871-4

19 Schievink WI, Schaid DJ. The prognosis of familial versus nonfamilia aneurysmal subarachnoid hemorrhage. Stroke 1996;27:340-1.

20 Ostbye T, Levy AR, Mayo NE. Hospitalization and case-fatality rates for subarachnoid hemorrhage in Canada from 1982 through 1991. The Canadian Collaborative Study Group of Stroke Hospitalizations. Stroke 1997;28:793-8.

21 Truelsen T, Bonita R, Duncan J, Anderson NE, Mee E. Changes in subarachnoid hemorrhage mortality, incidence, and case fatality in New Zealand between 1981-1983 and 1991-1993. Stroke 1998;29:2298-303.

22 Menghini VV, Brown-RDJ, Sicks JD, O'Fallon WM, Wiebers DO. Incidence and prevalence of intracranial aneurysms and hemorrhage in Olmsted County, Minnesota, 1965 to 1995. Neurology 1998:51:405-11.

23 Linn FH, Rinkel GJ, Algra A, van-Gijn J. Incidence of subarachnoid hemorrhage: role of region, year, and rate of computed tomography: a metaanalysis. Stroke 1996;27:625-9.

(Accepted 5 November 1999)

\title{
Extent of misclassification of death from Creutzfeldt-Jakob disease in England 1979-96: retrospective examination of clinical records
}

\author{
Azeem Majeed, Petra Lehmann, Liz Kirby, Richard Knight, Michel Coleman
}

\author{
Abstract \\ Objective To investigate the extent to which deaths \\ from Creutzfeldt-Jakob disease were misclassified \\ during 1979-96. \\ Design Structured review of clinical records based on \\ predetermined criteria to determine whether death \\ could have been due to sporadic or variant \\ Creutzfeldt-Jakob disease. \\ Setting 100 health authorities and 275 NHS trusts in \\ England. \\ Subjects 1485 people who died aged 15-44 years \\ from selected neurological disorders in England \\ during 1979-96. \\ Main outcome measure Cause of death. \\ Results The clinical records of 705 (48\%) subjects \\ were successfully traced. Tracing of clinical records \\ was highest in subjects who died during 1990-6. \\ There was sufficient information in the records of 640 \\ (91\%) of the 705 subjects to exclude Creutzfeldt-Jakob \\ disease as a cause of death. In $61(9 \%)$ subjects, there \\ was insufficient information to reach any conclusion \\ about the validity of the cause of death recorded on \\ the death certificate. The clinical records of four \\ subjects were examined further by the National
}

Creutzfeldt-Jakob Disease Surveillance Unit; none was thought to have died from Creutzfeldt-Jakob disease.

Conclusions No new cases of sporadic or variant Creutzfeldt-Jakob disease were detected in a sample of deaths most likely to have included misclassified cases. This suggests that the surveillance system is unlikely to have missed a significant number of cases among people aged 15-44 years. Hence, any rapid increase in the number of cases of variant Creutzfeldt-Jakob disease in this age group is likely to be real not artefactual.

\section{Introduction}

A national surveillance programme for CreutzfeldtJakob disease was started in the United Kingdom in May 1990. The primary aim of the programme was to detect any change in the epidemiology of the disease that might be attributable to bovine spongiform encephalopathy. ${ }^{1}$ In 1996, Will and colleagues reported 10 cases of a variant of Creutzfeldt-Jakob disease that was possibly associated with bovine spongiform encephalopathy. ${ }^{2}$ Speculation that bovine spongiform encephalopathy and Creutzfeldt-Jakob
Office for National Statistics, London SW1V 2QQ

Azeem Majeed medical epidemiologist Petra Lehmann research officer Liz Kirby research officer

National Creutzfeldt-Jakob Disease

Surveillance Unit, Western General Hospital, Edinburgh EH4 2XU

Richard Knight consultant neurologist

London School of Hygiene and Tropical Medicine, London WC1E 7HT

Michel P Coleman professor of epidemiology and vital statistics

continued over

BMJ 2000;320:145-7 